\title{
DE LA VIABILITÉ DU TEXTE AUTOMATIQUE DANS LE THÉÂTRE : S'IL VOUS PLAT̂T, I, 1
}

\author{
Mariana KunEŠOVÁ \\ Université d'Ostrava
}

\begin{abstract}
En): The study researches to what extent the main means of artistic expression of the first phase of surrealism, the automatic text, can be operational in drama, the genre which is essentially based on acceptable significations. Firstly, the characteristics of the automatic text are made precise as to stylistics and pragmatics (motivation), and confronted with motivation principles in a dialogue. Subsequently, the study develops an analysis of a scene included in one of the earliest surrealist plays, S'il vous plaît, by the duo Breton - Soupault, published in Les Champs magnétiques in 1920. The analysis focuses on the act I - a very short independent " play », and its scene 1, which should cover the exposition and the emergence of the conflict. Such functions seem difficult to make compatible with «l'automatisme psychique pur » (Breton) and are thus a challenge for closer observation.
\end{abstract}

Keywords (En): automatic text; theatre; surrealism; isotopy; motivation; Breton; Soupault

Mots-clés (Fr) : texte automatique ; théâtre ; surréalisme ; isotopie ; motivation ; Breton ; Soupault

\section{Remarques préliminaires}

On le sait : la première étape du surréalisme - où celui-ci aspire à devenir non une esthétique, mais un chemin de libération de l'individu - définit le mouvement naissant comme une pratique de la création automatique : « automatisme psychique pur par lequel on se propose d'exprimer [...] le fonctionnement réel de la pensée. [...] $\gg^{1}$ (BRETON, 1987 : 328). La question de l'ampleur avec laquelle les textes poétiques et en prose découlant de cette technique peuvent apporter un réseau de significations acceptables a été de nombreuses fois posée et étudiée. ${ }^{2}$ Or à ma connaissance, il n'en va pas de même pour le théâtre. Ainsi, le présent article se consacrera à la viabilité du texte automatique au sein de ce genre dans lequel, puisque la représentation scénique nécessite particulièrement l'adhésion immédiate du récepteur (et que pour celle-ci, des significations opérationnelles sont essentielles) la présence d'isotopies est de première importance.

Pour le corpus, j'opterai pour Les Champs magnétiques d'André Breton et Philippe Soupault. Ce recueil, publié en 1920, est né des premières expériences,

\footnotetext{
${ }^{1}$ Voici la légendaire définition dans sa totalité : «SURRÉALISME, n.m. Automatisme psychique pur par lequel on se propose d'exprimer, soit verbalement, soit par écrit, soit de toute autre manière, le fonctionnement réel de la pensée. Dictée de la pensée, en l'absence de tout contrôle exercé par la raison, en dehors de toute préoccupation esthétique ou morale. ENCYCL. Philos. Le surréalisme repose sur la croyance à la réalité supérieure de certaines formes d'associations négligées jusqu'à lui, à la toute-puissance du rêve, au jeu désintéressé de la pensée. Il tend à ruiner définitivement tous les autres mécanismes psychiques et à se substituer à eux dans la résolution des principaux problèmes de la vie. »

${ }^{2}$ Par exemple : RiffaterRe Michaël (1969) ; JenNy Laurent (1973) : La surréalité et ses signes narratifs », Poétique, n¹6, p. 499-513 ; Reverseau Anne (2007), Microlectures des textes automatiques surréalistes : complexité, simplicité et complications, Fabula-LhT, n 3, URL : http://www.fabula.org/lht/3/reverseau.html. 
intenses, des deux auteurs avec l'écriture automatique ; pour Breton, il s'agit de la première œuvre surréaliste (BRETON, 1952 : 64-65). Hormis les textes poétiques, il contient deux courtes pièces de théâtre, dont j'examinerai la première, S'il vous plaît.

Concernant l'objectif d'explorer un texte dramatique coécrit par Breton - dont le rejet du théâtre est devenu plus tard proverbial ${ }^{3}$ - ajoutons que, à l'époque de la gestation du surréalisme et pendant ses débuts, ce même Breton ne cachait pas son admiration pour les pouvoirs que l'écriture automatique attribue au dialogue. «C'est encore au dialogue que les formes du langage surréaliste s'adaptent le mieux », pose le Manifeste, chaque réplique étant "le tremplin à la pensée de celui qui écoute » (BRETON, 1962 : 46, 50). Breton n'est pas non plus opposé au geste théâtral. Il a participé en tant que comédien aux représentations Dada ainsi qu'à celles des «sketchs » des Champs $^{4}$; qu'il s'est hâté de porter à la scène dès leur parution (BÉHAR, 1979 : 236). Ceci montre que la dimension théâtrale de ces textes était pour Breton loin d'équivaloir à un simple effet de style.

Dans les lignes suivantes, je m'intéresserai d'abord aux spécificités d'ordre stylistique et pragmatique (motivation) du texte automatique, ainsi qu' aux principes de motivation dans un dialogue. Puis, je présenterai brièvement S'il vous plaît et analyserai les caractéristiques discursives de son acte I, scène 1. L'acte I, de fait, constitue une très courte pièce indépendante de 3 scènes; la première scène devrait donc permettre l'exposition et le surgissement du nœud. Or comment ces rôles peuvent-ils être procurés par «l'automatisme psychique pur»? Se pencher sur cette question me parait constituer un défi.

\section{Le texte automatique et ses spécificités dans le contexte du théâtre}

Dans l'objectif d'une analyse du texte automatique, la définition bretonienne ${ }^{5}-$ psychologisante, philosophisante et concernant le processus de l'écriture et ses buts, non le produit - mérite un prolongement. Ainsi, Michaël Riffaterre évoque des « chaîne[s] ininterrompue[s] d'associations verbales », décrochant de l'attitude « utilitaire » vis-à-vis du langage (RIFFATERRE, 1969: 46) ; et notamment du principe de coopération ajouterais-je. Libre de ce principe, l'association « automatique » peut s'intéresser à n'importe quel élément sémantique ou formel d'un énoncé précédent (voire du contexte situationnel), et le développer très librement, tant en créant des parallélismes formels qu'en prolongeant certains sèmes, dénotés ou connotés. Le résultat est un glissement isotopique et un changement de contextes souvent radical.

Dans les textes à forme dialoguée, lorsque la motivation découle de «l'énoncé précédent », celui-ci peut appartenir soit à la même réplique, soit à une réplique

\footnotetext{
${ }^{3}$ Breton a reproché au théâtre d'ignorer la spontanéité authentique puisqu'il nécessite des répétitions ; d'être rationaliste puisque découlant de la décision du comédien de revêtir un rôle ; d'appartenir au système social que lui, Breton, haïssait. D'où aussi, à la fin des années 1920, les «excommunications » du groupe surréaliste qui frappent Antonin Artaud et Roger Vitrac.

${ }^{4}$ En 1920, Breton joue dans La Première et La Deuxième aventure céleste de Monsieur Antipyrine de Tzara, Le Serin muet de Ribemont-Dessaignes et, avec Soupault, dans les deux pièces des Champs (BÉHAR, 1979 : 438, 426, 407).

${ }^{5}$ Voir la note 1.
} 
précédente. Dans le deuxième cas, les études théâtrales emploient souvent le terme de bouclage, introduit par Michel Vinaver. Ce terme mesure, hormis l'immédiateté de la motivation, sa qualité et sa clarté, ce qui intéressera la présente étude. Ainsi, M. Vinaver propose par exemple le couple bouclages serrés/lâches (b. serré : celui qui «par son contenu sémantique, mais aussi par son agencement formel s'ajuste à la réplique précédente »; VINAVER, $2000:$ 903), ou le non-bouclage (qui « succède à la réplique précédente sans rapport de sens ni de forme »; VINAVER, 2000 : 903). Afin de pouvoir distinguer aisément entre la motivation produite par une réplique indépendante et à l'intérieur d'une même réplique, cette étude fera également l'usage du concept de «bouclage».

Pour les besoins de mon analyse, j'ajouterai à la terminologie existante le «bouclage automatique » (bouclage par association verbale décrochant de l'attitude « utilitaire » vis-à-vis du langage). Logiquement, le texte choisi laisse supposer que les seuls bouclages automatiques y seront présents. Or une précaution est de mise : un tel dialogue, bien que voulu « automatique » par ses auteurs, peut ne pas consister entièrement en un «automatisme pur ». Et ce notamment puisqu'il s'agit d'un texte dramatique, qui par définition tente de créer un personnage et de développer son parcours. Un tel dialogue peut donc contenir des stratégies autres, bien qu'intuitives, comme des changements thématiques (non-bouclages), ou des bouclages à contextes éloignés, reliés cependant métaphoriquement (sortes de bouclages lâches).

\section{S'il vous plait ${ }^{6}$ et son « dialogue automatique »}

Dès la première lecture analytique de $S^{\prime}$ 'il vous plaît, deux constatations de taille se présentent. D'abord, contrairement à ce que l'on pourrait supposer, il ne s'agit pas que d'un dialogue à plusieurs voix. En effet la dimension d'action n'y est guère négligeable (surtout dans les actes II et IV), et témoigne d'un jeu avec les structures narratives $^{7}$ : chacun des 4 actes représente une très courte pièce indépendante, différant des autres par son genre, sa thématique et sa tonalité. Pratiquement toutes ces pièces instaurent l'attente d'un spectacle réaliste, et ce dès les indications de décor. Pour l'acte I : «Un salon à 5 heures du soir. Porte au fond. Fenêtres à droite et à gauche. Deux fauteuils. Un pouf. Une table basse. Une lampe. Glaces. » (p. 127). Chaque fable est bâtie sur une situation à contrepied de toute attente. Ainsi, l'acte I concerne un triangle amoureux étonnant : un mari appartenant à la classe aisée, jeune et attentionné pour son épouse ; celle-ci, folle d'un homme de loin plus âgé que son mari, «courbé, cheveux gris », qui la néglige.

Ensuite, l'apparence automatique des paroles ne concerne pas tous les dialogues. Elle s'observe surtout dans les actes les moins basés sur l'action, soit le I et le III, mais pas dans leur totalité. Dans I, il s'agit de la scène 1 et d'une partie de la dernière scène, 3 . Dans la scène 1 , la parole insolite met en place deux types

\footnotetext{
${ }^{6}$ Je me servirai de l'édition des Champs magnétiques parue chez Gallimard en 1971 (BRETON, SOUPAUlT, 1971), p. 127-161.

${ }^{7}$ Ce qui correspond aux caractéristiques du théâtre surréaliste tel quel proposées par Michel Corvin : expérimentation avec la parole automatique, mais aussi subversion des catégories narratives de base (et mise en œuvre des valeurs du surréalisme ; CORvIN, 1998 : 1579).
} 
de situations : un entretien énigmatique et une lutte verbale, qui seront interrogés dans les lignes qui suivent.

\subsection{Exposition : tête-à-tête énigmatique}

Le climat énigmatique caractérise la première moitié de la scène 1 , qui équivaut à l'exposition (p. 127-129).

L'exposition commence par l'extase des amants. Dès l'incipit, les deux personnages produisent des répliques énigmatiques que, dans certains cas, seul le développement de la séquence permet de décoder.

L'effet d'énigme découle surtout des bouclages à apparence d'automatismes, dont le nombre est assez important (un tiers), et qui sont répartis dans la totalité de la séquence. Observés de manière isolée, ces énoncés présentent des qualités poétiques ou quasi aphoristiques, mais leur signification est incertaine. En effet, ils relient des énoncés aux contextes fortement éloignés et à une ouverture de signification importante, dont le bouclage ne peut être décodé qu'avec une importante contribution du cotexte (parfois, après que plusieurs répliques successives ont concouru à l'éclaircissement). Ainsi, il s'agit d'une forme extrême du « bouclage lâche », que j'appellerai bouclage affaibli.

Le dialogue contient également une métaphore affaiblie qui ne fait pas partie d'un bouclage. Or de façon tout à fait surprenante, aucun développement véritablement automatique n'est présent ni dans les bouclages, ni à l'intérieur des répliques. En effet, l'impression d'insolite est due, mis à part les bouclages affaiblis, à l'importante présence du symbolisme traditionnel voire du lieu commun, mais étonnamment « revitalisé » par l'emploi de contextes et rapports inédits, parfois à la limite de la logique ou à apparence alogique, mais mettant en circulation une richesse de significations très justes. De telles stratégies dotent le symbolisme traditionnel d'un entourage à proprement parler hors du commun, d'où une force perlocutoire élevée. L'insolite ainsi produit augmente aussi en raison de la condensation substantive des énoncés : assez fréquente, elle peut être à la limite de la cassure isotopique. Le dialogue contient aussi des expressions littérales, d'un nombre assez restreint.

L'exposition consiste en six couples assertion/réplique voire question/réponse. J'observerai ce dialogue en m' arrêtant sur chacun de ces couples. ${ }^{8}$

1 PAUL : «Je t'aime. » (Long baiser.)

2 VALENTINE : «Un nuage de lait dans un verre de thé. »

La réplique 2 semble constituer un non-bouclage spectaculaire. Il s'agit bien d'une réaction à une déclaration d'amour, confirmée par le long baiser - situation à tel point évidente que la suite paraît claire d'avance. Or cette attente se voit fortement déçue, d'où un effet de déstabilisation renforcé par la condensation substantive de la réplique 2 , qui ne contient aucune marque d'investissement de la part d'un «je » (ou d'un autre agent). Un autre aspect immédiatement apparent de

\footnotetext{
${ }^{8}$ Pour une meilleure orientation dans le texte, j'indiquerai chaque réplique avec un chiffre.
} 
la réplique 2 se trouve dans ses importantes propriétés prosodiques : des parallélismes rythmiques pouvant rappeler deux hémistiches d'un alexandrin, et de riches allitérations.

Sur le plan du contenu, la réplique 2 fait apparaître un contraste de couleur, et probablement aussi de quantité (lait/thé) ; il est donc possible de s'essayer à des intersections sémiques avec la réplique 1 . Par exemple, l'amour est dans la grisaille de la vie aussi peu visible que le «nuage de lait dans un verre de thé ». Cependant une telle signification n'est pas tout à fait convaincante : qu'un personnage concerné par une déclaration d'amour fasse succéder à cet aveu une vérité générale paraît peu plausible. D'ailleurs aucun indice, ni dans l'énonciation ni dans la situation d'énonciation, n'assure que cette possible signification soit à privilégier. $\mathrm{Vu}$ l'étonnante distance des contextes, la valeur immédiate de la réplique 2 consiste avant tout dans ses qualités prosodiques et dans son effet d'énigme.

3 PAUL : «Quelle peine veux-tu que j'aie à choisir entre le passage des Tropiques et dès que tu ouvres les yeux ces aurores plus lointaines qui m'éblouissent? Le phosphore blanc des lèvres des autres femmes m'avait jusqu'ici rendu l'amour impossible. Incertain de te trouver, j'écoutais la pluie des chevelures heurter les vitres de ma paresse. Il faut avouer que je me suis laissé longtemps prendre aux trompeuses altercations de ce couple rigide : le réverbère et le ruisseau. »

4 VALENTINE : «Parle sans crainte. Ces mots que tu vas dire, je les connais, mais qu'importe ! Voici que notre vie monte lentement avec tes yeux qui me regardent et m'oublient. Tu vas encore me bercer de ce souviens-toi, te souviens-tu ? »

La réaction de Paul, contrairement à la réplique 2, emploie d'abord le symbolisme immédiatement décodable du lieu commun. Puis elle glisse du pôle de la clarté immédiate dans le symbolisme traditionnel revitalisé, et s'achève sur une expression ouverte, énigmatique.

Dans la première phrase (« Quelle peine veux-tu... aurores... qui m'éblouissent ?»), les lieux communs (passage des Tropiques, yeux comparés à des aurores) prolongent l'aveu de Paul de la réplique 1, en marquant une détente interprétative et en représentant ainsi un repère pour l'orientation dans le dialogue. Les lieux communs, pourtant, ont aussi une allure insolite. Ce qui est dû tant au voisinage avec la réplique précédente, énigmatique, qu'à la présence d'un qualificatif à apparence absurde : « choisir entre le passage des Tropiques et dès que tu ouvres les yeux ces aurores plus lointaines ${ }^{9}[\ldots] »$. Ce qualificatif mérite d'être appelé paradoxal, car son emploi découle d'une opposition au niveau de la signification (distance/proximité, les Tropiques étant effectivement éloignés davantage que les yeux de Valentine). Insolite, ce qualificatif parvient à signifier l'inaccessibilité de l'interlocutrice avec une force perlocutoire élevée.

La phrase 2 («Le phosphore blanc... ») affiche une image ouverte : « le phosphore blanc des lèvres », dont la signification semble de prime abord incertaine, puis alogique. L'expression suggère beauté et pureté (sèmes de lumière et blancheur), et ce avec une intensité exceptionnelle ( phrase telle quelle est négative ( «m'avait rendu... l'amour impossible »). Un effort d'interprétation accru permet de proposer une signification acceptable dans le

\footnotetext{
${ }^{9}$ Je soulignerai systématiquement les expressions sur lesquelles j'attirerai l'attention.
} 
contexte donné : lèvres pâles. Ce symbolisme, à nouveau paradoxal, car opérant l'opposition positif/négatif, est néanmoins dans le contexte d'une déclaration d'amour tout à fait opérationnel : il ne casse pas, mais au contraire, maintient, l'atmosphère de l'harmonie et de la perfection.

La phrase 3 («Incertain..., j'écoutais... aux vitres de ma paresse. ») attribue une allure hors du commun au symbolisme traditionnel de la métaphore filée, et ce au moyen d'un « gros plan » inédit, la synecdoque « ma paresse ». Cette image paraît à première vue alogique, car produisant une inversion du moi physique et du moi psychique (n'employant de ce dernier, en outre, qu'un seul trait). Dans le contexte donné, elle se révèle cependant un outil convenable, permettant de préciser la signification de la phrase : les appâts des autres femmes n’ont touché Paul que de manière superficielle et ne sont pas parvenus à le tirer de sa paresse. La synecdoque, donc, condense des images qui dans un dialogue habituel ne pourraient apparaître que de manière beaucoup plus « diluée » par des éléments accessoires, et qui disposeraient d'un impact perlocutoire moindre.

La dernière phrase (« Il faut avouer... ») entraîne par son contexte inattendu d'abord une incertitude d'interprétation : l'affirmation selon laquelle Paul s'est laissé « longtemps prendre aux trompeuses altercations de ce couple rigide : le réverbère et le ruisseau » constitue-t-elle un court-circuit isotopique ? Or la situation, un tête-à-tête faisant suite à une déclaration d'amour, moment chargé de signification, motiverait difficilement un tel gouffre sémantique. Ainsi, l'énoncé s'ouvre davantage au chemin interprétatif lancé par Paul même : « le réverbère et le ruisseau » en tant qu'images d'un « couple rigide », de l'organe masculin et du principe féminin. L'image donc évoquerait, comme la phrase précédente, les relations entretenues par Paul avant Valentine, et l'intention en serait d'alléger ce souvenir avec un mot d'humour (profitant du climat poétique de la réplique telle quelle, qui rend l'allusion sexuelle moins apparente). Lors de la représentation, une aide efficace à l'interprétation de cet étonnant énoncé pourrait découler du jeu de l'acteur. Celui-ci est susceptible de rendre la signification immédiate et évidente (avec le ton de la voix, l'emploi d'accessoires...), tout comme de laisser au spectateur un parfum d'énigme.

La réaction de Valentine (4), avec sa littéralité, puis son symbolisme aisément interprétable, exprimant un manque de confiance dans les paroles de son amant (« Tu vas encore me bercer de ce souviens-toi, te souviens-tu ? »), représente pour le décodage des significations une nouvelle détente. En même temps, elle apporte une précision importante quant à la situation en train de se dérouler : malgré le climat insolite créant l'impression de se retrouver dans un monde décroché du nôtre, on assiste à un germe de conflit banal des relations sentimentales. En d'autres termes, c'est bien «notre monde », de préoccupations « réalistes » que cette situation concerne.

Il est à noter que la phrase 3 («Voici que notre vie... ») revitalise une fois de plus le symbolisme traditionnel avec des rapports imprécis ou paraissant à la limite de l'acceptabilité logique : " notre vie monte lentement avec tes yeux qui me regardent » (ce qui signifie sans doute une certaine sensation de bonheur, mais peu convaincante). L'insolite de ces imprécisions va même jusqu'à revitaliser le 
symbolisme tout à fait traditionnel de la phrase suivante : « me bercer de ce souviens-toi $[\ldots] »$.

Parallèlement, la réplique 4 suggère, et ce avec un important retard, une interprétation opérationnelle de la réplique 2 (l'énigmatique réaction de Valentine à la déclaration d'amour : «Un nuage de lait dans un verre de thé. »). Celle-ci peut être appréhendée comme un constat, au moyen d'un bouclage affaibli, du peu de crédibilité de l'aveu de Paul. À nouveau, comme pour la réplique précédente de Paul (3), cette signification qui se dévoile à la lecture avec un tel temps de retard, pourrait, par le jeu de la comédienne, être procurée de manière immédiate et évidente (ou en maintenant une certaine dimension d'énigme).

5 PAUL : « Il faut se tenir à une certaine distance du mur pour éveiller l'écho. Avec ceux que nous aimons, l'espoir est d'arriver les bras tendus à entourer le tronc de cet arbre supraterrestre. »

6 VALENTINE : «Les mille et une nuits se fondent en une des nôtres. J'ai rêvé que nous nous noyions. »

La réaction de Paul (5) contraste avec la clarté interprétative de la réplique précédente. En intersection avec le posé dévoilant le manque de sincérité de Paul (4), la première phrase ( « Il faut se tenir... ») est lisible comme un bouclage affaibli affirmant qu'il faut, vis-à-vis de l'être aimé, maintenir une distance, de façon à ce que celui-ci s'attache à son partenaire au plus haut point. La deuxième phrase est décodable comme la nécessité de parvenir, en compagnie des êtres aimés, au contact avec un puissant élément transcendant (« cet arbre supraterrestre »). Ce qui, ensemble avec la première phrase de la réplique, revient à dire que ce n'est pas la fusion absolue avec un seul être qui relèverait des objectifs de Paul.

Ces significations ne sont pas décodables aisément. Afin qu'elles puissent être saisies dans la transposition scénique, cette fois-ci aussi, le jeu du comédien sera capital. Ajoutons néanmoins que le jeu de l'acteur, ici, ne devrait pas faire disparaître une certaine dose d'énigme poétique. C'est que les paroles de Paul semblent voulues telles, sans doute pour que leur signification véritable demeure cachée à Valentine.

Valentine, dans sa réaction (6), se laisse justement happer par la poésie qui l'amène à l'extase, laquelle en dit long sur l'intensité de ses sentiments pour Paul. Contrairement à son interlocuteur, elle use du symbolisme traditionnel, immédiatement déchiffrable : «Les mille et une nuits se fondent en une des nôtres. $[\ldots] »$.

7 PAUL : «Il y a longtemps que la charmante statue qui domine la tour Saint-Jacques a laissé tomber la couronne d'immortelles qu'elle tenait dans la main... Comment te plais-tu dans ton nouvel appartement? »

8 VALENTINE : «Le bureau de mon mari donne sur les jardins du Palais-Royal. »

La réaction de Paul (7) présente à nouveau des difficultés de décodage pour lequel, encore une fois, le contexte aura un rôle crucial. L'intersection avec le profil peu sincère de Paul fait apparaître cette réplique comme un bouclage affaibli, à signification cynique : «il y a longtemps que la relation avec Valentine ne représente plus quelque chose d'essentiel ». Cette signification est donc aussi cachée dans un 
voile poétique et énigmatique. Et à la représentation, la mesure dans laquelle cette signification transparaîtrait dépendrait du jeu du comédien.

Paul semble ne pas achever sa réflexion énigmatique et la remplace brusquement par une question littérale. La question, portant sur le nouvel appartement de Valentine, montre une fois de plus que le contexte situationnel n'est pas coupé d'un « univers réaliste »; au contraire, il semble s'apparenter à celui d'un drame bourgeois.

La réponse de Valentine (8), littérale aussi, présente en tant qu'information accessoire une circonstance essentielle : l'amoureuse folle de l'homme qui la néglige est une femme adultère. Pour le développement de la situation, cette information est essentielle ; c'est ici même que se situe le point culminant du caractère dramatique du dialogue. Ce pic est nourri tant par la lisibilité immédiate de la réplique que par sa brièveté. En outre, le motif de l'adultère confirme la similitude de la situation avec le genre du drame bourgeois. Et la réplique telle quelle, la similitude avec « notre monde »- car c'est bien un espace existant, parisien, qui est évoqué.

9 PAUL : «Ah oui ! Le jeu de barres. »

10 VALENTINE : «Méchant. Et ces miettes aux oiseaux : la solitude ? Les contrées de l'imagination sont d'un vaste !»

La réaction de Paul (9), vu son contexte apparemment incompatible avec celui de la réplique précédente, apparaît comme un non-bouclage absolu. Même si une telle réaction semble peu justifiée en tant que réponse à l'information que Paul a demandée, la réplique paraît résister à toute tentative de retrouver une signification opérationnelle. L'atmosphère d'énigme atteint ici son point culminant.

C'est enfin la réponse de Valentine (10), littérale, qui permet d'appréhender «le jeu de barres », comme un jeu par lequel Valentine échangerait son mari contre une autre distraction (ainsi, la réplique 9 se serait servie, comme plusieurs fois chez Paul déjà, du bouclage affaibli). Ajoutons que la présente réplique emploie, comme la précédente, la condensation substantive. Ce qui maintient le tempo élevé avec lequel apparaissent les significations, ainsi que, bien qu'il s'agisse d'une réplique littérale, un climat insolite. ${ }^{10}$

11 PAUL (surprenant dans la glace un de ses propres jeux de physionomie) : «C'est très justement que l'on a comparé certains regards à l'éclair : ils font apparaître les mêmes branches brisées, les mêmes jeunes filles blondes appuyées à des meubles noirs... Tu es plus belle qu'elles. »

12 VALENTINE : «Je sais. Tu aimes les châtaignes étincelantes qui se fondent dans mes cheveux. »

(Silence.)

Paul réagit (11), sans doute afin de ne pas prolonger le léger reproche de Valentine, par un changement de sujet en forme de songe, auquel se propose le

${ }^{10}$ Il revient à Georges Mounin d'avoir observé que les images surréalistes se succèdent avec une vitesse par excellence, « la vitesse maximum », réduisant les textes à un « essentiel poétique » (BRIOLET, 1984 : 55). 
contexte matériel d'énonciation ( "aperçoit dans le miroir un de ses propres jeux de physionomie »). Il évite donc de s'engager dans des réflexions sur la solitude de Valentine, mais ne brise pas le climat érotique : il incorpore dans son scénario des « jeunes filles blondes » et conclut sur un mot de galanterie, « Tu es plus belle qu'elles ». Le symbolisme employé est un symbolisme traditionnel revitalisé par des images d'apparence incompatible, mêlant des sèmes à valeur négative (thématique de la nature) et positive (celle de la femme). Il contient aussi des précisions inédites : des «branches brisées », et notamment de "jeunes filles blondes appuyées à des meubles noirs ».

La réponse de Valentine, avec son symbolisme traditionnel, fait comprendre que le mot galant a produit son effet. Le symbolisme apparaît ici également comme insolite, en raison du voisinage avec l'imaginaire inédit de la réplique précédente.

\section{Bilan}

L'exposition est bâtie sur le principe de coopération bien davantage que l'on n'aurait supposé de prime abord. Ce résultat tient tant à l'absence du dialogue automatique qu' au fait qu'aucun des personnages ne refuse de réagir aux propos de son interlocuteur (bien que ces réactions puissent être délicates à appréhender). De plus, l'exposition, malgré les paroles étonnantes et un nombre fort restreint d'indications d'action, remplit tout à fait ses objectifs : présenter les personnages, leurs caractéristiques de base et leur appartenance à un groupe (social), leurs désirs, objectifs ou besoins et ce qui les entrave, tout en donnant à entendre le genre auquel la pièce appartient. Tous ces objectifs sont atteints de manière dynamique et en captivant l'attention.

Le dialogue se sert efficacement d'un mouvement systématique énigme/clarté, incertitude/certitude. Ainsi, les bouclages affaiblis, par leur effet d'énigme et de suspense, renforcent le caractère dramatique des parties les plus sensibles : l'incipit ; la «présentation » d'un personnage - Paul ; la réaction de Paul à la réplique dévoilant que Valentine est mariée. Dans ces parties, les bouclages affaiblis voilent les formulations qui, si elles étaient plus explicites, contiendraient bien moins de drame. Et même : l'absence des éléments affaiblis installerait le dialogue dans un schéma habituel, quasi périmé - celui de la querelle d'un couple, où un personnage montre une intensité de sentiments moindre que l'autre. ${ }^{11}$ Ajoutons que la parole énigmatique caractérise systématiquement les posés qui sont de première importance pour la situation, y compris le manque d'authenticité des sentiments de Paul. Elle contribue aussi à dévoiler un trait important de Valentine : le fait de prendre une réplique énigmatique à contenu cynique pour une déclaration d'amour fait apparaître chez ce personnage une tendance à la naïveté.

\footnotetext{
${ }^{11}$ Les bouclages affaiblis, on l'a vu, ne créent pas l'impression que le dialogue soit coupé de l'univers « réaliste ». Ceci entre autres parce qu'ils sont interprétables comme ayant une justification logique : Valentine appréhenderait de formuler son manque de confiance dans les sentiments de Paul de manière trop brusque ; son interlocuteur souhaiterait ne pas donner à entendre clairement ses attitudes peu ménageantes pour Valentine.
} 
Pour rendre plus accessible la lisibilité des bouclages affaiblis, malaisée à la seule lecture, le jeu du comédien s'avère un outil puissant : tant pour suggérer une signification de manière pratiquement évidente que pour maintenir une brèche d'incertitude, dont l'épaisseur peut varier.

Contre-pôle des éléments affaiblis, le symbolisme traditionnel revitalisé et la littéralité ont le rôle important de repères immédiats pour l'interprétation, contrebalançant les effets de la parole hors de ses gonds et contribuant ainsi au dynamisme du dialogue. La littéralité est certes présente de manière minoritaire, or les énoncés concernés - surtout l'aveu initial et, au milieu du dialogue, le couple question/réponse dévoilant que Valentine est mariée - expriment des contenus essentiels qui déclenchent les moments dramatiquement les plus forts. Le rôle de ces énoncés (mis en valeur par leur brièveté) dans le dialogue est donc essentiel. En outre, la littéralité, renvoyant à plusieurs reprises à la situation sous sa forme matérielle, géographiquement ainsi que socialement « réaliste », confirme que c'est bien d'une fable campée dans «notre monde » qu'il est question.

Quant au contexte matériel de l'énonciation encore, rappelons que l'exposition se sert deux fois de didascalies. Présentes au début et immédiatement avant la fin du dialogue, elles attribuent à la situation un cadre à épaisseur matérielle sinon une vraisemblance plus élevée. Elles montrent en effet qu'il s'agit bel et bien de personnages en chair et en os qui agissent, et non de voix lâchées dans le vide, et stimulent l'imagination du lecteur (d'autant plus qu'une des didascalies exploite un détail spatial peu commun : « ... dans la glace un de ses propres jeux de physionomie »).

\subsection{Surgissement du conflit : lutte verbale}

Ce type de situation caractérise la deuxième moitié de la scène 1 (p. 129 -130). Ici, la soudaine inquiétude du retour du mari transforme le dialogue en une lutte verbale : Paul tente d'éviter le sujet que Valentine considère comme urgent - le besoin d'amour - tandis que son interlocutrice s'efforce de le réinstaller au centre de l'attention.

Le climat d'une lutte est dû surtout à un nombre élevé de changements thématiques. La plupart ont le caractère de ruptures-attaques : souvent un sujet ne se développe même pas car il se voit évincé par un autre, concurrent. L'intensité de cette lutte est dans deux cas renforcée par un procédé à effet encore plus déroutant : des esquives-attaques, se servant de bouclages automatiques. Ainsi, non seulement celui qui prend la parole évite un sujet qui lui est désagréable, mais il développe la réplique de l'interlocuteur d'une manière qui casse le principe de coopération. Parallèlement aux ruptures, les esquives- attaques sont des coups rapides, se limitant à une phrase.

Hormis la brièveté des répliques, c'est aussi la condensation substantive qui participe à l'atmosphère d'une lutte rapide. Le même rôle appartient à l'abondance d'ellipses interprétatives employées dans les bouclages.

Enfin, c'est l'imaginaire, sous une forme inédite ainsi que sous celle du symbolisme traditionnel, revitalisé ou non, qui sait renforcer le caractère déroutant des changements et des ruptures thématiques par des contextes d'association inattendus, porteurs d'une ironie mordante. 
Le climat de lutte se voit à plusieurs reprises pénétré de répliques pensives concernant le sujet de l'amour. Leur force perlocutoire est renforcée par l'imaginaire inédit aussi bien que traditionnel ; une réplique découle du bouclage automatique.

1 PAUL : «L'as-tu entendu entrer?»

2 VALENTINE : «La morale courante, on pense à l'eau courante. »

3 PAUL : «Le charme est dans cette chanson liquide admirable, l'épellation des enfants au catéchisme... Au besoin, de quoi parlez-vous? »

4 VALENTINE : « Une patience d'ange. J'ai une patience d'ange. Il louerait une villa, un pied-à-terre pour la saison. Beaucoup de lierre. Comme les autres hommes, il est tour à tour esclave de sa fatigue et de sa joie. » (Arrangeant un pli de sa robe.) «Ma robe te plaît?»

5 PAUL : «La boîte des bras à l'intérieur de peluche bleue. »

6 VALENTINE : «Amour. »

7 PAUL : «La chair ou les perles. Scaphandrier dans les ondes de cristal. Tout ce qui tient à un fil. »

8 VALENTINE : «Le paradis commence où bon nous semble. Le jour gris ardoise a des cornes d'autos bleues, la nuit on vole sur une palme argentée. »

9 PAUL : «Que fais-tu demain?»

10 VALENTINE : «Les grands magasins seront ouverts : la jeunesse de tant de femmes. »

11 PAUL : «À l'inspecteur qui se tient debout près de la porte : "L'ascenseur, monsieur, s'il vous plaît" ?»

12 VALENTINE : «Le sourire des vendeurs. Une tout autre coquetterie. »

Silence.

«À quoi penses-tu? »

13 PAUL : «La douceur de vie. Tout le monde s'en mêle. Les fils de la Vierge à la hauteur du visage des hommes, le chant des capitales. »

14 VALENTINE : «Tu ressembles à ces employés qui, à l'arrêt des trains, passent avec un marteau le long des roues. »

15 PAUL, distrait: « Je me suis souvent demandé quelle peut être en rapide et en amour la vitesse des mouches qui vont de la muraille arrière à la muraille avant du compartiment à couchette ou autre. » (Revenant brusquement à elle.) «Tu n'as pas froid? 》

16 VALENTINE : «Quelle heure est-il ?»

Silence.

«Paul, mon bonheur est doux comme les oiseaux affamés. Tu peux jouer en baissant les paupières ou en fermant les poings. Je consens à être désespérée. J'ai tellement pensé à toi depuis l'autre jour! »

\section{Bilan}

La totalité des structures discursives provoque efficacement le surgissement du conflit et le climat d'une intense lutte, entrecoupée de plusieurs réflexions songeuses au sujet de l'amour. Pour ce qui est du rôle de la plus importante inconvenance pragmatique, les bouclages automatiques (répliques $3,12,15$ ), il ne s'agit pas d'un moyen qui vise à créer une atmosphère insolite avant tout. L'insolite de ces bouclages ne brise pas la situation, mais au contraire, la développe : en esquivant une thématique désagréable et en attaquant l'interlocutrice $(3,15)$ et/ou en manifestant une distraction pensive $(12,15)$. L'acceptabilité des bouclages automatiques est due dans une mesure importante à leur contexte réaliste : d'une part, les deux esquives-attaques sont immédiatement suivies d'un énoncé tout à fait littéral, ce qui les ancre profondément dans la situation de l'énonciation. D'autre part, ce « réalisme » découle de l'état du personnage qui prend la parole, qui est 
dans un cas explicité (« distrait»: 15) et dans un cas implicite (réplique «songeuse »: 12).

Quant aux ruptures thématiques, celles-ci deviennent spectaculaires dans les trois dernières répliques, où le sujet lancé change quatre fois. Ces changements précipités, se terminant, après un silence d'hésitation, par l'abordage du thème essentiel pour Valentine, témoignent du fait que les autres sujets entamés ne représentaient qu'un recouvrement peu efficace mais justement de ce thème, considéré par Paul comme gênant. C'est aussi l'emploi du silence, chargé de signification et dramatiquement efficace, qui est à souligner (il en va de même pour l'autre moment de silence présent dans ce dialogue, en 12).

Parallèlement à l'exposition, les structures discursives précisent le caractère des deux personnages. Ainsi, Paul manifeste son cynisme, et ce dès la mention du rapport de Valentine envers son mari ( 3 : « Au besoin, de quoi parlez-vous ?»). La même attitude se présente dans la réaction à la réplique « Ma robe te plait ? » (4) : « La boîte des bras à l'intérieur de peluche bleue » (5). Valentine apparaît en revanche comme naïve, puisque considérant cette même réplique comme une déclaration d'amour poétique (6). Une certaine simplicité d'esprit et le caractère romanesque de Valentine sont aussi trahis par sa périphrase en 14, puis dans la dernière réplique par : «Je consens à être désespérée ». Or ce personnage n'est pas que doux et simple : une des répliques de Paul évoque l'aisance avec laquelle Valentine attire l'attention des autres hommes (11), ce que confirme l'intéressée (12). En outre, dans les structures discursives que ce personnage emploie, la naïveté n'est pas systématique, mais est contrebalancée par le symbolisme traditionnel à précisions inédites : «Le jour gris ardoise a des cornes d'autos bleues [...] $\gg(8)$, «Mon bonheur est doux comme les oiseaux affamés » (16). Valentine a donc un caractère dramatiquement bien plus porteur que si on la réduisait à une naïve soumise.

Les structures discursives précisent aussi l'attitude des deux personnages vis-àvis de l'amour, ce qui apporte de nouveaux éléments au profil de Paul. De façon surprenante, c'est Paul qui produit la première réplique pensive suite au mot « Amour » (6). Cette réaction, à allure inédite, interprète ce sentiment comme une beauté rare, à la fois sensuelle, pure et fragile, et comme une profondeur : « La chair ou les perles. Scaphandrier dans les ondes de cristal. Tout ce qui tient à un fil. » (7). Un peu plus tard cependant, Paul, pensif, confesse ironiquement : « La douceur de vie. Tout le monde s'en mêle. [...] (13). Ainsi, le personnage qui a souvent fait preuve de cynisme, entendrait l'amour comme une valeur d'exception, et hésiterait, du moins l'espace d'un instant, entre son acceptation et son rejet réduisant ce sentiment à une «douceur de vie » illusoire. La deuxième interprétation paraît l'emporter, à en juger par l'ironie criante de l'amour dans 15. Cependant, une certaine tendresse reste, vu que la réplique outrageuse se clôt par un soudain souci de Valentine : «Tu n'as pas froid ? Une telle complexité du personnage de Paul attribue à la scène un intérêt psychologique croissant. ${ }^{12}$

\footnotetext{
${ }^{12}$ Dans la dernière scène, 3 , la tentation de «la chair et les perles » verra une fin dramatique : suite à un geste profondément érotique de Valentine, avec lequel elle croit s'attacher Paul de manière inconditionnelle, celui-ci, dans un état de calme absolu, l'assassine - et reste sur place, alors qu'il est de plus en plus évident que le mari qui devait être loin, revient.
} 
Ajoutons enfin que le dialogue, à l'instar de la partie précédente, n'oublie pas de stimuler l'imagination au moyen d'interactions avec le contexte matériel de l'énonciation. Les didascalies, bien que toujours très rares, s'intéressent efficacement au détail («[...] un pli de sa robe »; «revenant brusquement à elle »).

\section{Conclusion}

L'analyse consacrée au « dialogue automatique » dans S'il vous plaît $(\mathrm{I}, 1)$ a confirmé l'hypothèse formulée dans la partie introductive : le dialogue ne consiste pas en un «automatisme [...] pur ». Ceci, entre autres, permet au dialogue de mettre en place une situation dramatique traditionnelle. Qui plus est, dans ce dialogue à l'allure profondément insolite, les développements automatiques sont quasiment absents. ${ }^{13}$ Ce qui revient à dire que l'insolite surréaliste peut découler de moyens bien moins radicaux parmi lesquels, dans le dialogue étudié, le symbolisme traditionne $\mathrm{l}^{14}$; « revitalisé » au moyen de contextes et rapports inédits, parfois paradoxaux, ou, dans une moindre mesure, sans modifications.

Même les inconvenances pragmatiques les plus malaisées à décoder - les bouclages automatiques et affaiblis - ne cassent pas la situation en train de se dérouler, mais la servent. À l'acceptabilité de ces bouclages contribuent aussi leurs intentions ou motivations, lisibles comme réalistes (voiler certains posés peu ménageants, esquiver une thématique désagréable, etc.). Ces bouclages, avec leurs effets d'énigme et de suspense, de rupture-attaque et d'esquive-attaque, ont un impact direct sur le dynamisme de la scène.

Le dialogue tel quel met en place une exposition et un surgissement du nœud efficaces. De plus, cette parole inédite, vivante au sens fort du mot, « recycle » les schémas dramatiques habituels (conflit entre deux amants dont l'un néglige l'autre), situations qui, si elles étaient traitées de manière traditionnelle se verraient vidées de l'essentiel de leur force perlocutoire. Dans le contexte de l'efficacité dramatique, il faut souligner l'importance du jeu des comédiens, susceptible de rendre plus accessibles les significations difficilement décodables à la seule lecture (mais aussi de maintenir, là où cela serait de mise, une dose d'énigme). Le dynamisme de la scène et ses pics dramatiques profitent également des didascalies, montrant souvent un grand détail qui stimule l'imagination, et d'un usage opérationnel des moments de silence.

Ainsi, la scène interrogée montre une dimension dramatique et théâtrale porteuse, davantage que l'on n'aurait eu tendance à le supposer. Au-delà d'une scène et d'un texte particuliers, il apparaît que le dialogue surréaliste, lorsque se

\footnotetext{
${ }^{13}$ Il est fort probable que d'autres textes dramatiques du surréalisme - la totalité créés de manière spontanée - disposent d'une dimension « automatique » différente (à commencer par les autres actes de $S$ 'il vous plaît). L'un de mes objectifs est de me pencher sur l'intérêt théâtral de ces textes dans mes travaux futurs.

${ }^{14}$ Il semble que la présence des images provenant du symbolisme traditionnel, à valeurs positives, bien que dans un contexte insolite, est propre de l'œuvre de Breton telle quelle. Voir à ce sujet par exemple ÉTIEMBLE René (1967), Breton? Un beau classique, N.R.F., 172, p. 845-846.
} 
destinant à la scène, sait devenir une « langue vivante ${ }^{15}$ et bâtir des situations à puissant effet.

\section{BIBLIOGRAPHIE}

BÉHAR Henri (1979), Le Théâtre dada et surréaliste, Paris, Gallimard.

BRETON André (1952), Entretiens, Paris, Gallimard.

ID. (1962), Manifestes du surréalisme, Paris, Pauvert.

ID. (1987), Euvres complètes I, Paris, Gallimard.

Breton André ; Soupault Philippe (1971), S'il vous plaît, in : Les Champs magnétiques, Paris, Gallimard, p. 127-161.

BRIOLET Daniel (1984), Le Langage poétique. De la linguistique à la logique du poème, Paris, Nathan.

CORVIN Michel (1998), Surréalisme, in : Dictionnaire encyclopédique du théâtre, Paris, Larousse - Bordas, p.1579.

RIFFATERRE Michaël (1969), La métaphore filée dans la poésie surréaliste, Langue française, 3, p. 46-60.

VINAVER Michel (2000), Écritures dramatiques, Paris, Actes Sud.

\footnotetext{
${ }^{15}$ Cette expression m'a été inspirée par le titre d'un stage pour comédiens tenu à Paris, au Théâtre de la Tempête, en 2008 : «Le vers racinien comme langue vivante » (dirigé par François Raffenaud).
} 